\title{
Genome variation in precision medicine
}

\author{
Precision medicine is a sufficiently imprecise term to cover cohort research in epidemiology as well as evidence- \\ based improvements in clinical delivery and informatics. We think it should continue to build and improve upon the \\ rigorous standards for measurement of genome sequence variation.
}

$\mathrm{P}$ recision medicine takes into account measurements of individual variation. That defines the discipline as a broad tent, including existing evidence-based medicine initiatives, targeted treatments in oncology and much of preclinical research. Within the remit of precision medicine, there are all kinds of measurements and initiatives, but some are more likely to be useful than others. Genetic variation is inborn and therefore one of the more frequently identified causal factors in disease, and it is one of the most general and relatively inexpensive forms of individual variation to measure. Biomarkers will likely be superseded by mechanistic insights anchored to genome variation, regardless of whether they are based upon correlates of DNA and histone modifications or measures of gene expression. Without underlying sequence, structural variation and three-dimensional architecture, it is very hard to establish what these markers denote, whether predisposition, exposure, cell type or disease state. For this reason, we think it is a good idea to design these studies around genome sequence and not in isolation.

We have held a "Genome Variation in Precision Medicine" Nature conference for the past six years in China (from its origins as a GWAS workshop in Hefei in 2011 to the most recent meeting 27-29 May in Shenzhen), and "The Genomics of Common Diseases" Nature conference is in its tenth anniversary year (25-28 September in Baltimore). Consequently, we have considerable interest in what precision medicine is taken to mean and where the field is moving. Personalized medicine is the wrong term for precision medicine and creates an unsupported impression of the value of the individual experience. Individuals are best treated as members of groups to standardize practice for teaching, economics, safety and the statistical validity of evidence. Translational medicine is research that advances the first clinical trial in humans. Of course, we do not advocate the use of research results in medicine without a properly designed, ethically sound clinical trial.

Medical informatics and bioinformatics need to merge to do research on current records of practice and delivery. Precision medicine will work when the discovery, clinical and delivery arms of the medical establishment integrate their research to provide evidence for constant and iterative improvement of practice. So it is gratifying to see elec- tronic medical records that are somewhat laborious for clinical staff to record made very useful in research for establishing commonalities in genetic predisposition to different medical conditions via phenomewide association studies (PheWAS; Nat. Biotechnol. 31, 1102-1110, 2013). These commonalities in predisposition are investigated further on pages 707, 709 and 811 of this issue. This work shows that, although association studies have limited usefulness in establishing causation, it is possible to begin to investigate the dependency of one diagnosis on another from their underlying SNP associations.

Genomic epidemiology has now moved to a larger scale and greater integration with health records and national health provider infrastructures via Precision Medicine Initiatives in the United States and China and the Genomics England 100,000 Genomes Project in the UK. In line with this expansion, key steps toward the successful use of genomic information are published in this issue. Two methods are presented for phasing genotypes across entire biobanks of hundreds of thousands of individuals (pages 811 and 817 ). These methods should be broadly applicable. Another report on page 740 is both broad in scope and deep in detail: Xuejun Zhang and colleagues present the sequence of the human major histocompatibility complex of 20,635 Han Chinese individuals, recording the genome region that makes the greatest genetic contribution to multiple diseases and predispositions. This work also potentially shows the diversity and distribution of organ transplant donors and recipients across China, making it a centerpiece for future health research.

We are keen to see sound medical research emerge in good time from precision medicine based on genomics and urge patience rather than hype. There will be few 'quick-win' solutions to current medical problems, and bypassing the advances made in genomics rather than building logically upon them would be a mistake. In the short term, translational success should be gauged as increases in the number of phase 1 clinical trials, decreases in the costs and rate of failure of subsequent trials, as well as improvements in the outcomes and economics of existing therapies. Ultimately, the epidemiological objective of replacing treatments with early diagnosis and prevention will need to be documented, lest the advances of genomic medicine be absorbed into the economics of medicine as usual. 